\title{
A qualitative systematic review of head-to-head randomized controlled trials of oral analgesics in neuropathic pain
}

\author{
C Peter N Watson MD FRCPC ${ }^{1}$, lan Gilron MD FRCPC ${ }^{2}$, Jana Sawynok PhD ${ }^{3}$
}

CPN Watson, I Gilron, J Sawynok. A qualitative systematic review of head-to-head randomized controlled trials of oral analgesics in neuropathic pain. Pain Res Manage 2010;15(3):147-157.

BACKGROUND: Neuropathic pain (NP) encompasses many difficultto-treat disorders. There are few head-to-head, comparative, randomized controlled trials (RCTs) of drugs for NP in different analgesic categories, or of different drugs within a category, despite many placebo-controlled RCTs for individual agents. Well-designed head-to-head comparative trials are an effective way to determine the relative efficacy and safety of a new drug.

OBJECTIVE: To perform a systematic review of head-to-head RCTs of oral analgesics in NP.

METHODS: A systematic review of RCTs involving NP patients was performed, of which head-to-head comparative trials were selected. Reference lists from published systematic reviews were searched. These studies were rated according to the Jadad scale for quality.

RESULTS AND CONCLUSIONS: Twenty-seven such trials were identified. Seventeen were comparisons of different analgesics, and 10 were of different drugs within an analgesic class. Important information was obtained about the relative efficacy and safety of drugs in different categories and within a category. Some significant differences between active treatments were reported. Trial inadequacies were identified. More and improved head-to-head RCTs are needed to inform clinical choices.

Key Words: Head-to-head trials; Neuropathic pain; Randomized controlled trials; Systematic review

\begin{abstract}
"Food and Drug Administration (FDA) regulations should require that new drugs be compared not just with placebos but with old drugs for the same conditions. Approval would depend on whether the new drug adds something useful in terms of greater effects, greater safety, fewer side effects or substantially greater convenience...If I could choose only one of the reforms I am suggesting it would be this one. There is an ethical issue here too. It is wrong to compare a new drug with a placebo if there is an effective drug already on the market." (Marcia Angell [1])

"Interpretation of the results of trials of treatments for neuropathic pain would be greatly facilitated by the inclusion of active comparators with well established efficacy." (Katz et al [2])
\end{abstract}

$\mathrm{H}$ ead-to-head randomized controlled trials (RCTs) are critical in all areas of clinical medicine (1), and neuropathic pain

\section{Revue systématique qualitative des essais randomisés contrôlés comparant directement des analgésiques oraux dans la douleur neuropathique}

HISTORIQUE : La douleur neuropathique (DN) englobe de nombreuses entités difficiles à traiter. Peu d'essais randomisés et contrôlés (ERC) ont comparé directement des analgésiques de différentes catégories ou d'une même catégorie dans la DN, malgré l'existence de nombreux ERC avec témoins sous placebo ayant porté sur certains agents individuels. Les essais de comparaison directe bien conçus permettent de déterminer clairement l'efficacité et l'innocuité relatives d'un nouveau médicament.

OBJECTIF : Réaliser une revue systématique des ERC de comparaison directe entre des analgésiques oraux dans la DN.

MÉTHODE : Les auteurs ont procédé à une revue systématique des ERC ayant regroupé des patients souffrant de DN et ont recensé les essais de comparaison directe. Ils ont aussi consulté les listes bibliographiques des revues systématiques publiées et ont classé les études selon l'échelle de qualité Jadad.

RÉSULTATS ET CONCLUSIONS : Les auteurs ont ainsi relevé 27 essais dont dix-sept comparaient des analgésiques différents et dix comparaient divers agents d'une même classe d'analgésiques. Ils en ont tiré une information importante sur l'efficacité et l'innocuité relatives des médicaments d'une même catégorie ou de catégories différentes. Certaines distinctions significatives entre les traitements actifs ont été observées. On a relevé des lacunes dans les essais. Pour faire des choix cliniques éclairés, il faudra procéder à d'autres ERC de comparaison directe plus rigoureusement conçus.

(NP) is no exception (2). NP has been defined as pain initiated or caused by a primary lesion or dysfunction in the nervous system (3). (An amended definition of NP has been proposed recently [4] but has not been accepted by the International Association for the Study of Pain.) When the lesion or dysfunction occurs in the peripheral nervous system, it is termed peripheral NP (PNP); when it occurs in the central nervous system, it is termed central pain. Central pain disorders may occur after stroke, spinal cord injury, traumatic brain injury, multiple sclerosis and syringomyelia. There are many PNP disorders (Table 1 and Figure 1 [5]). Most clinical research on drugs useful for NP has been performed in patients with postherpetic neuralgia and painful diabetic neuropathy, but it is reasonable that a similar treatment approach may be applied to patients with other PNP disorders (except trigeminal neuralgia, which is a different and unique form of NP) and central disorders based on putative common mechanisms. Clinical experience indicates that some conditions, such as central pain and lumbar radiculopathies (the most common form of PNP)

${ }^{1}$ University of Toronto, Toronto; ${ }^{2}$ Queen's University, Kingston, Ontario; ${ }^{3}$ Dalhousie University, Halifax, Nova Scotia

Correspondence and reprints: Dr C Peter N Watson, 1 Sir Williams Lane, Toronto, Ontario M9A 1T8. Telephone 416-239-3494,

fax 416-239-6365, e-mail peter.watson@utoronto.ca 
TABLE 1

Categories of peripheral and central neuropathic pain

Peripheral

Osteoarthritis/disc disease with nerve root pain (usually C5 and C6, and L5 and S1)

Postherpetic neuralgia (may have a central component)

Painful neuropathies (diabetes, alcohol/nutritional, HIV, etc)

Cancer-associated neuropathic pain

Phantom limb pain

Nerve trauma (causalgia)

Incisional neuralgias (post-thoracotomy, postmastectomy, etc)

Brachial plexus avulsion (likely a central component)

Neuropathic facial pain exclusive of trigeminal neuralgia

Trigeminal neuralgia*, glossopharyngeal neuralgia

Central

Central poststroke pain

Spinal cord injury pain

Traumatic brain injury

Multiple sclerosis

Syringomyelia

*Trigeminal neuralgia is a unique and common form of neuropathic pain with a differing medical and surgical approach from other forms of neuropathic pain (except the rare condition of glossopharyngeal neuralgia)

(Figure 1), are generally less responsive than others. Some peripherally generated problems can have a central component (postherpetic neuralgia and brachial plexus avulsion) and central sensitization may occur with other PNP disorders, making the distinction between peripheral and central pain states less clear. NP has different qualities, often in the same patient. Steady - often burning - pain, shock-like jabbing pain and pain from touch (allodynia) may all occur, indicating possible different pain mechanisms for such qualities and supporting a mechanism-based approach (6).

Thirty years ago, there was little science behind the few drugs used for NP conditions. Amitriptyline, phenothiazines and the combination of amitriptyline and fluphenazine were widely used based on uncontrolled and observational studies (7-10). With the evolution of trial methodology and clinical research, current data suggest that the major categories of drugs shown to be useful for NP by RCTs are antidepressants, anticonvulsants, opioids and the emerging field of cannabinoids. There are many such studies in NP (11); most are RCTs that show a moderate effect of a drug compared with placebo. The utility of these drugs in clinical practice based on these RCTs that is, the external validity or generalizability of these findings - has been a neglected issue until recently (12). Thus, most RCTs do not provide measures of clinical meaningfulness, such as effect size, number needed to treat (NNT) (13) and number needed to harm (NNH), which makes comparisons between specific agents difficult (12). NNT and NNH values have been generated for some of these RCTs by others (11).

Since the first head-to-head RCT discussed here (published 20 years ago) (14), there have been few subsequent trials of this nature in NP comparing drugs in different analgesic classes or different drugs within an analgesic class. A recent review regarding algorithm development (11) identified only 13 headto-head RCTs of 105 NP RCTs, but provided useful comparative data in their absence in the form of NNT and $\mathrm{NNH}$ values (Table 2). The advantage of head-to-head RCTs in NP is that they enable the clinician, struggling with the problem of the

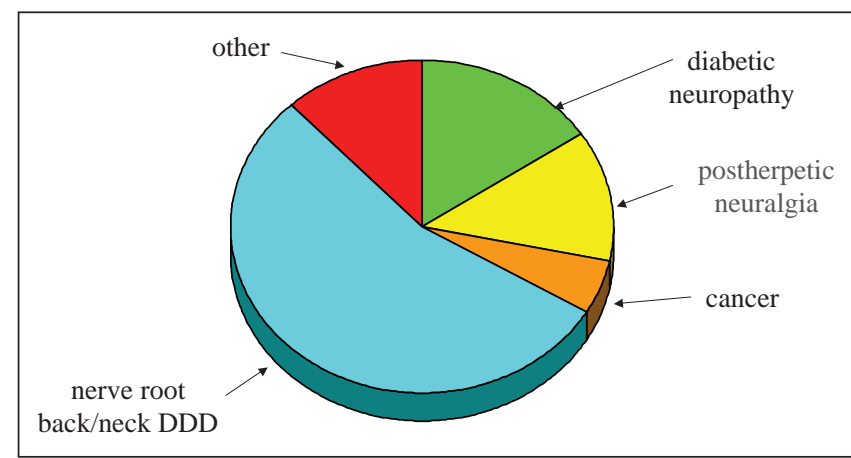

Figure 1) Prevalence of some forms of peripheral neuropathic pain. DDD Degenerative disc disease. Adapted from Irving (5)

TABLE 2

Number needed to treat for neuropathic pain randomized controlled trials of tricyclic antidepressants, selective serotonin/norepinephrine reuptake inhibitors (SNRIs), opioids, gabapentinoids and cannabinoids

\begin{tabular}{llll}
\hline Drug & $\begin{array}{l}\text { Postherpetic } \\
\text { neuralgia }\end{array}$ & $\begin{array}{l}\text { Diabetic } \\
\text { neuropathy }\end{array}$ & Other neuropathic pain \\
\hline All tricyclics & $2.3,2.3,2.1$ & $3.0,2.4,3.4$ & $2-5$ \\
Venlafaxine & 4.5 & & 5.2 \\
Imipramine & & $5.0,4.1$ & 2.7 \\
Duloxetine & & 3.7 & $5.1,3.8$ \\
Gabapentin & $3.2,5.0$ & & 4.2 \\
Pregabalin & 3.4 & 2.6 & \\
Oxycodone & 2.5 & & $3.5,3.7$ for multiple \\
Cannabinoids & & & $\begin{array}{l}\text { sclerosis-related } \\
\text { neuropathic pain }\end{array}$ \\
& & & 3.9 \\
\hline Tramadol & 4.76 & 4.3 &
\end{tabular}

Data from reference 11

most appropriate drug selection, to know whether a new drug is equal to, noninferior to or better than a standard therapy (such as a tricyclic antidepressant [TCA]) and whether there are differences in adverse effects. The only other way to determine relative efficacy and safety is to compare measures such as NNT and NNH data from placebo-controlled trials. However, these measures usually compare studies with differences in experimental design, number of subjects, trial duration, inclusion criteria, pain intensity, outcome measures and data analyses; consequently, they can only be, at best, an approximate comparative measure.

Head-to-head trials may also reveal data about pain mechanisms, in that one drug may work in a particular subgroup. For example, it is possible that one of the drugs compared may relieve predominantly burning pain, electric shock-like pain or allodynia, revealing a mechanism-based difference in drug responsivity (6). Therapeutically, this may be valuable in indicating drugs for combination therapy targeting different pain mechanisms. Further subgroups, such as age, sex, race and diagnostic categories, may also reveal differences between drugs.

The purpose of the present article is to review the data obtained from head-to-head RCTs of oral pharmacotherapeutic agents in chronic noncancer NP to determine whether there is clear evidence for drug selection choices. 


\section{METHODS}

A qualitative systematic review of head-to-head RCTs was performed by the primary author (CPNW) using the following terms: (pain OR painful OR analgesia OR nociceptive $O R$ antinociceptive OR antinociception) AND (neuropathic OR neuropathy $O R$ neuralgia $O R$ nerve $O R$ radiculopathy $O R$ radicular OR sciatica OR polyneuropathy) AND (Randomized Controlled Trial) searching PubMed, Medline and the Cochrane Databases for RCTs and systematic reviews of oral analgesics (antidepressants, anticonvulsants, opioids and cannabinoids) in chronic noncancer NP of three months duration or longer. The main focus was on trials in adults published in English from 1966 to 2009. In addition, the reference lists of retrieved articles and the FDA website (www.fda.gov) were searched.

Trials were evaluated according to the quality criteria of Jadad et al (15). To be included, trials were required to score at least $3 / 5$ on this rating scale to ensure a standard of quality; this required randomization and double-blinded conditions, with a control group and accounting for withdrawals. A maximum score of 5 additionally indicated that the RCT described methods of blinding and randomization. Measures were sought in each trial, such as effect size, percentage of patients with $50 \%$ or greater improvement, NNT, NNH and number needed to quit (NNQ), as a means of determining clinical meaningfulness. The search excluded non-English publications, trials that compared topical agents, intravenous studies, acute NP conditions, NP with cancer, and the NP conditions of trigeminal neuralgia and complex regional pain syndrome.

\section{RESULTS}

\section{General}

Table 3 lists excluded trials $(\mathrm{n}=10)(16-25)$ and summarizes reasons for their exclusion. The included RCTs are presented in Tables 4 and 5. Twenty-seven such trials were identified. Table 4 documents 17 head-to-head trials of different analgesics in NP (14,26-41). Table 5 lists 10 comparative trials involving different drugs within an analgesic class (42-51). Of this latter group, all were comparisons of antidepressants. Nineteen of the 27 trials (70\%) were conducted in postherpetic neuralgia, painful diabetic neuropathy or a combination of both conditions. The minimum duration of pain in the trials was three months in all except two trials $(28,37)$.

Twenty-one trials were of a crossover design $(14,27,29,31$ $33,35-39,41-50)$ and six were of a parallel design $(26,28,30,34,40,51)$. The median number of patients in the crossover trials was 37 (range 11 to 96) and the number of patients in each of the six parallel trials was $n=49(30), n=76$ (34), $\mathrm{n}=145$ (28), $\mathrm{n}=246$ (26), $\mathrm{n}=338$ (40) and $\mathrm{n}=47$ (51). All except one (26) were nonindustry-sponsored trials. None of these comparative trials showed a newer drug (gabapentin, $\mathrm{n}=6$; pregabalin, $\mathrm{n}=1$; venlafaxine, $\mathrm{n}=2$; and lamotrigine, $\mathrm{n}=1$ ) to be superior in relieving pain to the older active comparator. Some measure of clinical meaningfulness was available from 18 trials, but NNT figures were only reported in three trials $(32,39,50)$. Quality of life was not an outcome measure in many of these RCTs, but was included in a few studies $(26,27,31-33,35,38,41)$. Nineteen trials included a placebo control $(14,26-28,30-33,35,36,39-46,48,50)$.

\section{TABLE 3}

Excluded comparative head-to-head randomized controlled trials

\begin{tabular}{|c|c|}
\hline Study & Reason for exclusion \\
\hline $\begin{array}{l}\text { Watson and Evans, } 1985 \text { (16); amitriptyline } \\
\text { versus zimelidine in postherpetic neuralgia }\end{array}$ & Nonblinded, nonrandomized \\
\hline $\begin{array}{l}\text { Gomez-Perez et al, } 1985 \text { (17); nortriptyline } \\
\text { and fluphenazine in diabetic neuropathy }\end{array}$ & $\begin{array}{l}\text { Not head-to-head, used } \\
\text { combination of } \\
\text { nortriptyline/fluphenazine } \\
\text { versus placebo }\end{array}$ \\
\hline $\begin{array}{l}\text { Ventafridda et al, } 1988 \text { (18); trazodone } \\
\text { versus amitriptyline in deafferentation pain }\end{array}$ & NP with cancer \\
\hline $\begin{array}{l}\text { Harke et al, } 2001 \text { (19); carbamazepine } \\
\text { versus morphine in NP pretreated with } \\
\text { spinal stimulation }\end{array}$ & $\begin{array}{l}\text { Series of two placebo- } \\
\text { controlled trials } \\
\text { (carbamazepine versus } \\
\text { placebo and morphine } \\
\text { versus placebo), possible } \\
\text { order effect (population in } \\
\text { phase } 2 \text { not same as } \\
\text { phase } 1 \text { ) }\end{array}$ \\
\hline $\begin{array}{l}\text { Pandey et al, } 2005 \text { (20); gabapentin versus } \\
\text { carbamazepine in Guillain-Barré syndrome }\end{array}$ & $\begin{array}{l}\text { Acute pain, likely both } \\
\text { inflammatory and } \\
\text { neuropathic }\end{array}$ \\
\hline $\begin{array}{l}\text { Galer et al, } 2005 \text { (21); morphine versus } \\
\text { morphine/dextromethorphan in chronic } \\
\text { noncancer non-NP }\end{array}$ & Non-NP \\
\hline $\begin{array}{l}\text { Lynch et al, } 2005 \text { (22); amitriptyline versus } \\
\text { ketamine in NP syndromes }\end{array}$ & Topical preparations \\
\hline $\begin{array}{l}\text { Keskinbora et al, } 2007 \text { (23); gabapentin } \\
\text { versus opioids in neuropathic cancer pain }\end{array}$ & Neuropathic cancer pain \\
\hline $\begin{array}{l}\text { Rowbotham et al, } 1991 \text { (24); lidocaine versus } \\
\text { morphine in postherpetic neuralgia }\end{array}$ & $\begin{array}{l}\text { Intravenous study, short } \\
\text { term }\end{array}$ \\
\hline $\begin{array}{l}\text { Kok-Yuen et al, } 2008 \text { (25); topical amitriptyline } \\
\text { versus lidocaine in NP }\end{array}$ & Topical preparations \\
\hline
\end{tabular}

Comparative trials of different analgesics for NP

Comparative trials of different analgesics for NP are summarized in Table 4. Most of the 17 comparative trials of different analgesics for NP compared a potential analgesic drug with a TCA, except for the comparisons of venlafaxine with gabapentin (31), gabapentin with morphine (33), nabilone with dihydrocodeine (38), morphine with mexiletine (39) and gabapentin with oxycodone (40).

The 1999 diabetic neuropathy study by Morello et al (29) comparing gabapentin with amitriptyline found no significant difference (NSD) in either pain relief or side effects between drugs. This study has been criticized because of the low dose of gabapentin used compared with the placebo-controlled trials in postherpetic neuralgia and painful diabetic neuropathy (52), and also the lack of placebo control. However, the degree of pain relief achieved (52\% with gabapentin and $67 \%$ with amitriptyline) suggests that the statistically equal effect did not mean that both drugs were ineffective.

Results from an industry-sponsored trial comparing amitriptyline with pregabalin have been posted on the FDA website (26); however, complete details of this trial are not available for review. This unpublished Pfizer Protocol 1008-040 (www.accessdata.fda.gov/scripts/cder/drugsatfda) (26) apparently studied painful diabetic neuropathy and compared placebo, amitriptyline $75 \mathrm{mg}$ and pregabalin $600 \mathrm{mg}$ in a parallel design. An FDA review found that both drugs produced a 2.8point reduction in pain intensity (versus 1.8 with placebo), but 
TABLE 4

Comparative trials of different potential analgesics in neuropathic pain $(n=17)$

\begin{tabular}{|c|c|c|c|c|c|c|}
\hline $\begin{array}{l}\text { Study, condition, } \\
\text { duration of pain }\end{array}$ & $\begin{array}{l}\text { Jadad } \\
\text { score }\end{array}$ & Drugs, daily dose & $\begin{array}{l}\text { Completed/ } \\
\text { randomized, } \mathbf{n}\end{array}$ & $\begin{array}{l}\text { Effect, } \\
\text { yes/no }\end{array}$ & Comment & $\begin{array}{l}\text { Clinical meaningfulness } \\
\text { and quality of life }\end{array}$ \\
\hline $\begin{array}{l}\text { Max et al, } 1988 \\
\text { (14), postherpetic } \\
\text { neuralgia, } \\
19 \text { months (mean) }\end{array}$ & 3 & $\begin{array}{l}\text { AT mean } 65 \text { mg/day, } \\
\text { range } 12.5-150 \mathrm{mg} / \text { day; } \\
\text { lorazepam range } \\
0.5-6 \mathrm{mg} / \text { day; placebo } \\
\text { (lactose) }\end{array}$ & $41 / 58$ & $\begin{array}{l}\text { Yes (SSD) (AT); } \\
\text { no (lorazepam) }\end{array}$ & $\begin{array}{l}\text { Crossover design; AT effective } \\
\text { but not as an antidepressant; } \\
\text { lorazepam ineffective }\end{array}$ & $\begin{array}{l}47 \% \text { moderate or greater relief } \\
\text { with AT versus } 15 \% \text { with } \\
\text { lorazepam and } 16 \% \text { with } \\
\text { placebo }\end{array}$ \\
\hline $\begin{array}{l}\text { Leijon and Boivie, } \\
1989 \text { (27), central } \\
\text { poststroke pain, }\end{array}$ & 4 & $\begin{array}{l}\text { AT } 75 \text { mg; CBZ } \\
\text { 200-800 mg/day; } \\
\text { placebo }\end{array}$ & $14 / 15$ & $\begin{array}{l}\text { Yes (SSD) (AT); } \\
\text { no (NST) (CBZ) }\end{array}$ & $\begin{array}{l}\text { Crossover - } 3 \text { phase; no AT } \\
\text { antidepressant effect; CBZ } \\
\text { not effective }\end{array}$ & $\begin{array}{l}67 \% \text { improved on } \mathrm{AT} \text { versus } 36 \% \\
\text { with } \mathrm{CBZ} \text {; NNQ = } 0\end{array}$ \\
\hline
\end{tabular}

longer

Kieburtz et al, 1998

(28), HIV

neuropathy,

2 weeks or longer

$4 \quad$ AT $25-100 \mathrm{mg} / \mathrm{day}$;

mexiletine 150-300 mg/

day; active placebo

$\begin{array}{ll}\text { 126/145 (total); } & \text { No effect of any } \\ 39 \text { (AT); } 44 \quad \text { drug } \\ \text { (mexiletine); } \\ 43 \text { (placebo) }\end{array}$ $\begin{array}{ccccc}\text { Morello et al, } 1999 & 4 & \text { AT mean } 59 \mathrm{mg} ; & 19 / 25(\mathrm{AT}) ; & \text { Yes (AT); } \\ \text { (29), diabetic } & & \text { GP mean } 1565 \mathrm{mg} & 19 / 25(\mathrm{GP}) & \text { yes (GP) }\end{array}$

neuropathy,

3 months

\section{Graff-Radford et al, 2000 (30), postherpetic neuralgia, 6 months \\ Simpson, 2001 (31), diabetic neuropathy, 3 months or longer}

\begin{tabular}{|c|c|c|}
\hline 5 & $\begin{array}{l}\text { AT } 12.5-200 \text { mg/day; } \\
\text { fluphenazine } 1-3 \text { mg/day; } \\
\text { AT } 75 \text { mg per day/ } \\
\text { fluphenazine } 1 \text { mg } 3 \text { times } \\
\text { per day; active placebo }\end{array}$ & $49 / 49$ \\
\hline 4 & $\begin{array}{l}\text { GP up to } 3600 \text { mg/day; } \\
\text { venlafaxine up to } \\
75 \text { mg/day; placebo }\end{array}$ & $\begin{array}{c}\text { 11/11 in RCT } \\
\text { phase; GP/ } \\
\text { venlafaxine, } \\
\text { n=5; GP/ } \\
\text { placebo, n=6 }\end{array}$ \\
\hline
\end{tabular}

Raja et al, 2002

(32), postherpetic neuralgia,

3 months
Gilron et al, 2005

(33), postherpetic neuralgia/diabetic neuropathy,

3 months

Chandra et al, 2006 (34); postherpetic neuralgia, longer than 8 weeks

Pfizer protocol 1008040 (26), diabetic neuropathy (refer to FDA website), unpublished after one year
$5 \quad$ NT mean 89 mg/day, $\quad$ 44/76 range 40-140 mg/day; or DES mean 63 mg/day; morphine mean $91 \mathrm{mg} /$ day, range $15-225 \mathrm{mg} /$ day; or methadone mean 15 mg/day; placebo

5 GP (up to $3200 \mathrm{mg} /$ day); morphine (up to $120 \mathrm{mg} /$ day); both GP (up to $2400 \mathrm{mg} /$ day) and morphine (up to $60 \mathrm{mg} /$ day); lorazepam placebo (1.6 mg/day)

5 GP up to $2700 \mathrm{mg} /$ day versus NT up to 75 mg/day; no placebo

3 AT $75 \mathrm{mg} /$ day fixed dose; pregabalin 600 mg/day; placebo
Yes (SSD) (AT); no (NSD) (fluphenazine); yes (SSD) (AT/ fluphenazine)

Yes (SSD)

3 parts to study GP versus
placebo, then nonresponders
enter crossover RCT of

11 subjects comparing GP + venlafaxine with GP + placebo; final phase was an uncontrolled study with venlafaxine added to GP nonresponders; SSD for some SF-36 scales

Yes (SSD) (NT); Crossover design; both NNT for opioids $=2.7$ and NNT for yes (SSD) (DES); yes (SSD) (morphine); yes (SSD) (methadone); no (placebo)

No (NSD) (GP); yes (SSD) (morphine); tricyclics and opioids effective; NST favoured opioids

tricyclics $=4.0$; Multidimensional Pain Inventory showed no difference in overall activity or pain-related interference in daily activity

$67 \%$ moderate or greater relief with AT; $52 \%$ moderate or greater relief with GP; $\mathrm{NNQ}=2$ for each drug

No result reported

n head-to-head RCT part of study, 3 subjects much/ moderately improved with GP/ venlafaxine versus 1 on GP/ placebo; SF-36 score improved with GP/venlafaxine yes (SSD) (GP/ analgesia with lower doses of morphine); each no (placebo)

No (NSD)

Parallel design; NSD in pain; more dose-limiting side effects with NT; dose high to start with (in our view) - NT at $50 \mathrm{mg}$

Yes (SSD) (AT); Parallel design; AT but not no (NSD) (pregabalin) pregabalin superior to placebo
30\% with placebo; AT significant for PPI of SF-MPQ, PGIC and CGIC; SF-36 results for both groups better than placebo 
TABLE 4 - CONTINUED

Comparative trials of different potential analgesics in neuropathic pain $(n=17)$

\begin{tabular}{|c|c|c|c|c|c|c|}
\hline $\begin{array}{l}\text { Study, condition, } \\
\text { duration of pain }\end{array}$ & $\begin{array}{l}\text { Jadad } \\
\text { score }\end{array}$ & Drugs, daily dose & $\begin{array}{l}\text { Completed/ } \\
\text { randomized, } \mathbf{n}\end{array}$ & $\begin{array}{l}\text { Effect, } \\
\text { yes/no }\end{array}$ & Comment & $\begin{array}{l}\text { Clinical meaningfulness } \\
\text { and quality of life }\end{array}$ \\
\hline $\begin{array}{l}\text { Khoromi et al, } 2007 \\
\text { (35), lumbar root } \\
\text { pain and chronic } \\
\text { sciatica, } 3 \text { months }\end{array}$ & 5 & $\begin{array}{l}\text { Morphine mean } 62 \mathrm{mg} / \text { day, } \\
\text { range } 15-90 \mathrm{mg} / \text { day; NT } \\
\text { mean } 84 \mathrm{mg} / \text { day, range } \\
25-100 \mathrm{mg} / \text { day; } \\
\text { combination, } 49 \mathrm{mg} / \text { day } \\
\text { morphine }+55 \mathrm{mg} / \text { day NT } \\
\text { (means); placebo }\end{array}$ & $28 / 55$ & $\begin{array}{l}\text { No (NSD) } \\
\text { (morphine); } \\
\text { no (NSD) (NT); } \\
\text { no (NSD) } \\
\text { (combination) }\end{array}$ & $\begin{array}{l}\text { Crossover design } \times 4 \text { phases; } \\
\text { no effect; high dropout rate } \\
\text { "suggests resistant problem" }\end{array}$ & $\begin{array}{l}\text { Moderate or greater relief for } \\
\text { morphine } 42 \%, \text { NT } 40 \%, \\
\text { combination } 67 \% \text { and placebo } \\
37 \% ; \text { NNH for morphine = } 10, \\
\text { NT = } 30 \text { and combination = 11; } \\
\text { SF-36 showed carryover and } \\
\text { period effects. No difference in } \\
\text { phase I analysis }\end{array}$ \\
\hline $\begin{array}{l}\text { Rintala et al, } 2007 \\
\text { (36), spinal cord } \\
\text { injury, } 6 \text { months or } \\
\text { longer }\end{array}$ & 5 & $\begin{array}{l}\text { AT } 150 \text { mg/day (max); GP } \\
3600 \text { mg/day (max); } \\
\text { diphenhydramine (active } \\
\text { placebo) } 75 \text { mg/day }\end{array}$ & $22 / 38$ & Yes (SSD) & $\begin{array}{l}\text { Crossover design } \times 3 \text { phases } \\
\text { with high scores for } \\
\text { depression; AT more effective } \\
\text { than diphenhydramine and } \\
\text { GP = diphenhydramine }\end{array}$ & $\begin{array}{l}\text { In high depression score group, } \\
30 \% \text { relief in } 62.5 \% \text { on AT, } \\
12.5 \% \text { on GP and } 25 \% \text { on } \\
\text { diphenhydramine; no quality of } \\
\text { life assessment }\end{array}$ \\
\hline $\begin{array}{l}\text { Jose et al, } 2007 \\
\text { (37), diabetic } \\
\text { neuropathy, } \\
1 \text { month }\end{array}$ & 4 & $\begin{array}{l}\text { Lamotrigine } 50-200 \mathrm{mg} / \mathrm{day} ; \\
\text { AT } 10-50 \mathrm{mg} / \mathrm{day} ; \\
\text { no placebo arm }\end{array}$ & $46 / 75$ & No (NSD) & $\begin{array}{l}\text { Crossover design; pain duration } \\
\text { only } 1 \text { month; may have } \\
\text { underdosed patients on AT }\end{array}$ & $\begin{array}{l}\text { Few differences in efficacy, fewer } \\
\text { side effects with lamotrigine; } \\
50 \% \text { improvement in } 41 \% \text { on } \\
\text { lamotrigine and in } 28 \% \text { on AT }\end{array}$ \\
\hline $\begin{array}{l}\text { Wu et al, } 2008 \text { (39), } \\
\text { postamputation } \\
\text { pain, } 6 \text { months or } \\
\text { longer }\end{array}$ & 5 & $\begin{array}{l}\text { Morphine mean } 112 \text { mg/day, } \\
\text { range } 15-180 \mathrm{mg} / \text { day; } \\
\text { mexiletine mean } \\
933 \mathrm{mg} / \mathrm{day} \text {, range } \\
300-1200 \mathrm{mg} / \text { day; } \\
\text { placebo }\end{array}$ & $56 / 60$ & $\begin{array}{l}\text { Yes (SSD) } \\
\text { (morphine); } \\
\text { no (NSD) } \\
\text { (mexiletine) }\end{array}$ & Crossover design & $\begin{array}{l}\text { NNT morphine }=5.6(50 \% \text { or } \\
\text { more); NNT morphine }=4.5 \\
(30 \% \text { or more })\end{array}$ \\
\hline $\begin{array}{l}\text { Gilron et al, } 2009 \\
\text { (41), neuropathic } \\
\text { pain (diabetic } \\
\text { neuropathy and } \\
\text { postherpetic } \\
\text { neuralgia), } \\
6 \text { months or } \\
\text { longer }\end{array}$ & 5 & $\begin{array}{l}\text { GP (target } 3600 \text { mg/day, } \\
\text { mean max } 2433 \mathrm{mg} / \text { day } \\
\text { in monotherapy versus } \\
2180 \mathrm{mg} / \text { day in } \\
\text { combination); NT (target } \\
100 \mathrm{mg} \text { per day, max } \\
\text { tolerated } 61.6 \mathrm{mg} \text { in } \\
\text { monotherapy versus } \\
51.1 \text { mg in combination) }\end{array}$ & $45 / 56$ & $\begin{array}{l}\text { Combination } \\
\text { therapy more } \\
\text { effective than } \\
\text { monotherapy }\end{array}$ & $\begin{array}{l}\text { Crossover design; not clear } \\
\text { whether effect is additive or } \\
\text { synergistic; no serious adverse } \\
\text { effects; no difference in } \\
\text { adverse effects except less } \\
\text { dry mouth with GP alone and } \\
\text { less inability to concentrate } \\
\text { with NT }\end{array}$ & $\begin{array}{l}\text { At least moderate relief: } 65 \% \text { on } \\
\text { GP, } 76 \% \text { on NT and } 84 \% \text { on } \\
\text { combination (NSD). SF-36: } \\
\text { Higher vitality score with } \\
\text { combination and GP versus NT; } \\
\text { weak evidence (P=0.057) that } \\
\text { combination better than } \\
\text { monotherapy }\end{array}$ \\
\hline
\end{tabular}

AT Amitriptyline; CBZ Carbamazepine; CGIC Clinician's global indication of change; DES Desipramine; FDA Food and Drug Administration; GP Gabapentin; max Maximum; NNH Number needed to harm; NNQ Number needed to quit; NNT Number needed to treat; NSD No significant difference; NST Nonsignificant trend; NT Nortriptyline; PGIC Patient's global indication of change; PPI Present pain index; RCT Randomized controlled trial; SF-36 Short-Form 36 Health Survey; SF-MPQ Short-Form McGill Pain Questionnaire; SSD Statistically significant difference

the reduction with pregabalin was not significantly different (the starting pain intensity in the pregabalin group was 0.5 points higher than in the amitriptyline group). In this trial, amitriptyline, but not pregabalin, was superior to placebo. The FDA review goes on to say that the above results came from a less conservative 'last observation carried forward' analysis but, with the more conservative 'baseline observation carried forward' analysis, neither pregabalin nor amitriptyline differed statistically from placebo with respect to mean pain intensity at the end point.

The 2000 placebo-controlled RCT by Graff-Radford et al (30) for postherpetic neuralgia compared amitriptyline with the phenothiazine fluphenazine, and the combination of the two drugs, and found NSD in pain with fluphenazine used 
TABLE 5

Comparative trials of different antidepressants in neuropathic pain $(n=10)$

\begin{tabular}{|c|c|c|c|c|c|c|}
\hline $\begin{array}{l}\text { Study, condition, } \\
\text { duration of pain }\end{array}$ & $\begin{array}{l}\text { Jadad } \\
\text { score }\end{array}$ & Drugs, daily dose & $\begin{array}{l}\text { Completed/ } \\
\text { randomized, n }\end{array}$ & Effect, yes/no & Comment & $\begin{array}{l}\text { Clinical meaningfulness } \\
\text { and quality of life }\end{array}$ \\
\hline $\begin{array}{l}\text { Panerai et al, } 1990 \\
\text { (42), central pain: } \\
\text { postherpetic } \\
\text { neuralgia, } \\
\text { phantom limb and } \\
\text { causalqia. }\end{array}$ & 3 & $\begin{array}{l}\text { Clomipramine } 25-100 \text { mg/day; } \\
\text { NT 25-100 mg/day; placebo }\end{array}$ & $24 / 39$ & $\begin{array}{l}\text { Yes (SSD) } \\
\text { (clomipramine); } \\
\text { yes (SSD) (NT) }\end{array}$ & $\begin{array}{l}\text { Crossover design; clomipramine } \\
\text { more effective (SSD) than NT; } \\
\text { no antidepressant effect; } \\
\text { conditions studied actually of } \\
\text { peripheral origin }\end{array}$ & Not stated \\
\hline
\end{tabular}

causalgia,

20.6 months

(mean)

Sindrup et al, 1990

(43), diabetic

neuropathy, 1 year

4 Paroxetine $40 \mathrm{mg}$ (fixed dose); 19/29

imipramine 50-75 mg;

placebo

\begin{tabular}{|c|c|c|}
\hline $\begin{array}{l}\text { Yes (SSD) } \\
\text { (paroxetine); } \\
\text { yes (SSD) } \\
\text { (imipramine) }\end{array}$ & $\begin{array}{l}\text { Crossover design; effect of S } \\
\text { drug (paroxetine) but imipramine } \\
\text { (serotonin/noradrenaline) more } \\
\text { effective (SSD); no } \\
\text { antidepressant effect; fewer } \\
\text { adverse effects with paroxetine }\end{array}$ & Not stated \\
\hline $\begin{array}{l}\text { Yes (SSD) } \\
\text { (clomipramine); } \\
\text { no (NSD) (DES); } \\
\text { no (placebo) }\end{array}$ & $\begin{array}{l}\text { Crossover design; clomipramine } \\
\text { (S) but not DES }(\mathrm{N}) \text { effect on } \\
\text { pain }\end{array}$ & Not stated \\
\hline
\end{tabular}

\section{Sindrup et al, 1990 \\ (44), diabetic \\ neuropathy \\ symptoms for \\ 1-20 years \\ Sindrup et al, 1992 \\ (45), diabetic \\ neuropathy \\ symptoms for \\ 1-11 years \\ Max et al, 1992 \\ (46), diabetic \\ neuropathy \\ 3 months}

Watson et al, 1992

(47), postherpetic

neuralgia,

3 months
3 Clomipramine 50-75 mg; DES 50-100 mg; placebo

4 Mianserin $60 \mathrm{mg} /$ day (fixed dose); imipramine 50-75 mg/day; placebo

3 AT mean $105 \mathrm{mg}$, range 12.5-150 mg; DES mean $111 \mathrm{mg}$, range $12.5-150 \mathrm{mg}$; fluoxetine mean $40 \mathrm{mg}$; placebo (benztropine 0.5-1.5 mg/day)

4 AT $100 \mathrm{mg}$ (37.5-150 mg/day); M 100 mg (50-150 mg/day); no placebo
$19 / 26$

$18 / 22$ no (placebo)

No (mianserin); yes (SSD)

(imipramine)

38/54 (AT); 38/54 (DES); $46 / 54$

(fluoxetine); 46/54 (placebo)

$32 / 35$ no (NSD) (fluoxetine)
Yes (SSD) (M) imipramine (noradrenaline + diabetic neuropathy, 14 = other neuropathy

\section{$31 / 33$}

(49), postherpetic neuralgia,

3 months

Sindrup et al, 2003 (50), painful neuropathy,

6 months

Rowbotham et al, 2005 (51), TCAnaive postherpetic neuralgia,

3 months

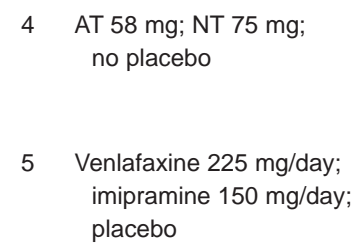

5 Venlafaxine 225 mg/day; imipramine 150 mg/day; placebo

$29 / 40$

$38 / 47$; fluoxetine $60 \mathrm{mg}$; no placebo

\section{3/15 (DES);} 15/17 (AT); $10 / 15$ (fluoxetine)
Crossover design, 3 phases; serotonin) effect but no mianserin effect (weakly $\mathrm{N}$, serotonin blockade)

Yes (SSD) (AT); 2 RCTs with 2 period crossover yes (SSD) (DES); studies; fluoxetine (S) not effective; $A T(\mathrm{~S}+\mathrm{N})=\operatorname{DES}(\mathrm{N})$; suggested $\mathrm{N}$ mechanism of action

Crossover design; $M(N)$ less effective than AT (S+N) (SSD); no antidepressant effect; some responded better to $M$, some to AT

Yes (SSD) (AT); yes (SSD) (M); no (placebo)

Not stated

$74 \%$ moderate or greater relief with AT versus $61 \%$ with DES and versus placebo $40 \%$; NSD between DES and AT

$47 \%$ had mild/no pain on AT and 38\% had mild/no pain on $\mathrm{M}$; good/ excellent (50\% or more), AT $=50 \%, M=18 \%$. No difference in disability ratings

Crossover, 3 phase; AT $(\mathrm{S}+\mathrm{N}) \quad 20 \%$ per reduction in $63 \%$ more effective than M (S) (SSD) AT, 50\% M and 22\% but both better than placebo; no placebo antidepressant effect; all pain qualities relieved; some had better pain relief on AT, some with $\mathrm{M}$

Yes (SSD) (AT); yes (SSD) (NT)

Crossover design; equal effects; 50\% improved less severe side effects with NT (SSD)

substantially on both drugs. No difference in disability ratings

Yes (SSD) (venlafaxine); yes (SSD) (imipramine)

Crossover, 3 ways; no difference NNT for the TCA in primary end point but more had complete, good or moderate relief with imipramine (SSD); number of adverse effects same except for type

Yes (SSD) (DES); Parallel design; TCA-naive yes (SSD) (AT); yes (SSD) patients; intent to treat showed NSD in daily pain intensity or pain relief with all 3 drugs imipramine was 2.7 and for the SNRI venlafaxine was 5.2; complete, good or moderate relief more common with imipramine No difference in daily pain intensity or relief (NSD) but moderate or greater relief in 9/17 (53\%) with AT, 12/17 (80\%) with DES and 5/15 (30\%) with fluoxetine (SSD); best clinically meaningful relief with $\mathrm{N}$ drug (DES)

AT Amitriptyline; CBZ Carbamazepine; DES Desipramine; M Maprotiline; N Noradrenergic; NNT Number needed to treat; NSD No significant difference; NST Nonsignificant trend; NT Nortriptyline; RCT Randomized controlled trial; S Serotonergic; SNRI Selective serotonin/norepinephrine reuptake inhibitor; SSD Statistically significant difference; TCA Tricyclic antidepressant 
alone or in combination with amitriptyline; however, it found a statistically significant difference in pain with amitriptyline. This was an important trial because this drug combination was one of the original treatments for NP $(9,10)$ and the combination of fluphenazine and nortriptyline was previously shown to be more effective than placebo (17). It is apparent from this RCT that amitriptyline is the analgesic component and, furthermore, that adding fluphenazine increased sedation without adding any analgesic benefit.

In 2007, Khoromi et al (35) studied chronic sciatic pain with placebo, morphine, nortriptyline and a combination of these two drugs, and found NSD with any of the treatments. This trial used between $15 \mathrm{mg}$ and $90 \mathrm{mg}$ of morphine per day with flexible dosing to maximal tolerable dose. Our own experience $(53,54)$ indicates that this may be an inadequate dose of morphine to alleviate this type of refractory NP, which may explain the lack of efficacy of opioids in sciatic pain in this RCT.

Gilron et al compared gabapentin, morphine and the two drugs in combination in one trial (33) and then compared nortriptyline, gabapentin and the two drugs in combination in a subsequent trial (41). In both of these trials, the two-drug combination demonstrated superior efficacy to each single agent. However, NSDs were observed between either of the single agents.

\section{Comparative trials of different drugs within an analgesic class used for NP}

Table 5 summarizes comparative RCTs of different drugs within an analgesic class. The 10 studies (42-51) listed in Table 5 are all head-to-head RCTs of different antidepressants conducted usually with the aims of discovering the most effective drugs in this class, and potentially to inform their mechanism of action. No trials of different drugs within other classes of analgesics for NP such as opioids, gabapentinoids (gabapentin and pregabalin) or cannabinoids met our criteria.

\section{General summary}

Several general observations can be made on the basis of these head-to-head studies. Of the RCTs comparing different potential analgesics, there is no evidence supporting the efficacy of the benzodiazepine lorazepam (14), the phenothiazine fluphenazine (30) or the sodium channel-blocking agents mexiletine (39) and carbamazepine (27) for NP (trigeminal neuralgia was excluded). There is no evidence for the superiority of gabapentinoids over TCAs either regarding pain or adverse effects, although the nature of the latter differs with the two agents $(26,29,36)$. There are nonsignificant trends suggesting the superiority of opioids over TCAs and gabapentinoids $(32,33)$.

With regard to antidepressant comparisons, the TCAs amitriptyline, nortriptyline, desipramine and imipramine do not appear to differ in analgesic efficacy. The noradrenergic TCA desipramine is at least as effective as amitriptyline in treating diabetic neuropathy and postherpetic neuralgia $(46,51)$. There is some indication that the noradrenergic antidepressant maprotiline, which has a tetracyclic structure, is less effective than amitriptyline. This has been shown by two studies $(47,48)$ that were similar in design and covered two different conditions - postherpetic neuralgia and painful neuropathy. Unlike imipramine, the nontricyclic agent mianserin was not efficacious (45). There is some evidence that the serotonergic drug clomipramine may be more effective than imipramine and desipramine $(42,44)$ and that the selective serotonin reuptake inhibitor (SSRI) paroxetine may be effective (43); however, these studies were small, may have been inadequately powered, did not state clinical meaningfulness and could have been vulnerable to type 1 error. There is no good evidence for the effectiveness of the SSRI fluoxetine in treating NP $(46,51)$. The preponderance of evidence from these RCTs is supportive of the greater efficacy of noradrenergic and noradrenergic/serotonergic TCAs in NP. The selective serotonin/norepinephrine reuptake inhibitor venlafaxine appears inferior to imipramine for meaningful relief (50). There is some evidence of individual variability in responsivity when individual antidepressants are compared using head-to-head RCTs (47-49), arguing for sequential trials of different antidepressants in individuals.

\section{DISCUSSION}

There are a limited number of head-to-head comparative RCTs of analgesics in NP published between 1988 and 2009. Some of those reviewed here were quite small and may have inadequate statistical power. Within our search criteria there were 17 studies of drugs in different analgesic categories and 10 of analgesics within a category, which were comparisons of different antidepressant drugs. There were seven RCTs using a gabapentin comparator, one using pregabalin, two using a selective serotonin/norepinephrine reuptake inhibitor antidepressant (venlafaxine) and one using the anticonvulsant lamotrigine. No consistent superiority of the latter drugs was found. There were no studies comparing different opioids, gabapentinoids or cannabinoids. All published RCTs were nonindustry funded. One industry-funded study submitted to the FDA (26) has not been published. Most head-to-head RCTs used a crossover design. Most NP RCTs were performed in postherpetic neuralgia patients, painful diabetic neuropathy patients or both, and this may limit their external validity (generalizability) to other NP conditions (12). There are limited data regarding clinical meaningfulness in many of these comparative trials, which is also problematic for determining their external validity (12).

\section{Comparative RCTs of different analgesics}

From the limited data in these head-to-head RCTs of different analgesics in NP, there is no evidence supporting the utility of the benzodiazepine lorazepam (14), the anticonvulsants carbamazepine (27) and lamotrigine (37), or the phenothiazine fluphenazine (in contrast to the favourable effect of the TCA amitriptyline [30]). Also, the sodium channel blocker mexiletine was ineffective compared with morphine for chronic postamputation pain (39). Two studies showed no effect of mexiletine or the comparator drug amitriptyline in HIV neuropathy (28), and no effect of nortriptyline or morphine in sciatica (35). These latter two results raise questions about the generalizability of research in NP, in which $80 \%$ of the studies are performed in postherpetic neuralgia and diabetic neuropathy (12). There appears to be no good evidence supporting the analgesic superiority of the gabapentinoids (gabapentin and pregabalin) over TCAs $(29,34)$ in these RCTs; amitriptyline was found to be superior in two studies $(26,36)$. Combinations of gabapentin added to morphine (33) and 


\section{TABLE 6 \\ Potential mechanisms of the analgesic action of antidepressants}

Serotonin potentiation by inhibition of reuptake

Noradrenaline potentiation by inhibition of reuptake

Combined noradrenaline/serotonin potentiation

Dopamine potentiation

Opioid-mediated actions

$\mathrm{Na}^{+}$channel blockade

$\mathrm{K}^{+}$channel activation

$\mathrm{Ca}^{2+}$ channel inhibition

Adenosine release

$\mathrm{N}$-methyl-D-aspartate receptor blockade

$\mathrm{GABA}_{\mathrm{B}}$ potentiation

Substance $P$ reduction

Prostaglandin reduction

Data from reference 59. GABA Gamma-aminobutyric acid

oxycodone added to gabapentin (40) resulted in a $20 \%$ and $33 \%$ increase in analgesia, respectively.

These comparative RCTs of different analgesics indicate efficacy within three broad classes of agents for NP - antidepressants, opioids and gabapentinoids. The structures of these agents are diverse. The receptors for opioids, and their location and mechanisms of action, have received considerable attention and are reasonably well characterized (55). The pharmacodynamics of gabapentinoids (gabapentin and pregabalin), a more recently available class of drugs, focus on the alpha-2-delta subunit of $\mathrm{Ca}^{2+}$ channels and interactions with descending noradrenergic monoamine systems $(56,57)$. Ironically, despite being the oldest and most-studied drugs for $\mathrm{NP}$, the analgesic antidepressants are incompletely understood from a pharmacodynamic perspective. Antidepressants have been well characterized in terms of their ability to inhibit noradrenaline and serotonin reuptake (58), but antidepressants as a class of drugs have a multiplicity of pharmacological actions that potentially contribute to pain modulation (Table 6) (59). The focus on amine mechanisms for explaining analgesia by antidepressants reflects their actions in relieving depression (the biogenic amine hypothesis of depression), as well as the involvement of noradrenergic and serotonergic systems descending from the brain stem into the dorsal horn of the spinal cord, in regulating pain signals as they enter the central nervous system. It is important to recognize that, while depression is common in patients with chronic pain (60), and while depression and chronic pain share mechanisms (61), pain relief with antidepressants is independent of antidepressant actions $(14,62)$; this dissociation has been recognized since the efficacy of antidepressants for NP pain was clearly established (63). Several lines of investigation, including the use of amine receptor antagonists and gene deletion approaches, indicate the requirement of noradrenergic and serotonergic systems for antinociception by antidepressants in several preclinical models $(59,64)$. However, several experimental lines of evidence indicate that antidepressants also interact with other mechanisms that contribute to their ability to modulate pain signalling (59).

\section{Comparative RCTs of antidepressants}

Comparative RCTs of different antidepressant agents generally indicate that drugs that potentiate noradrenaline and serotonin, or predominantly noradrenergic mechanisms, are more effective than serotonergic agents (such as the SSRIs), which are less or even not effective. The presence of both facilitatory and inhibitory descending serotonergic systems could be an important reason for this (64). There is some evidence that the more noradrenergic, weaker serotonergic TCAs nortriptyline (49) and desipramine $(46,51)$ are equal to or more effective than the dual noradrenergic/serotonergic agent amitriptyline. Other data from two RCTs using the tetracyclic comparator antidepressant maprotiline, which is more noradrenergic than nortriptyline and desipramine, showed it to be less effective than amitriptyline $(47,48)$. Thus, some serotonin augmentation appears to be important but the optimal proportion may not have been found yet. In keeping with this, the strongly serotonergic/weakly noradrenergic reuptake inhibitor venlafaxine appears to provide less clinically meaningful relief than the noradrenergic/serotonergic TCA imipramine (50). However, some comparative trials have also produced data that differs from these general observations because the serotonergic TCA clomipramine and the SSRI paroxetine have been shown to be effective in some instances $(42-44)$. One trial showing clomipramine to be superior to nortriptyline in central pain (42) actually investigated the peripherally generated pain disorders of postherpetic neuralgia, phantom pain and causalgia; this profile of indications differs from many other trials. These RCTs involved small numbers of patients and the clinical meaningfulness of the results was not clear.

There may be more than one pain mechanism in an individual and between individuals, and multiple mechanisms may contribute to analgesia. Both TCAs (and opioids) are indiscriminate in relieving steady pain, jabbing pain and allodynia $(47,49)$. To date, clear mechanism-based treatments have been elusive, except perhaps for carbamazepine and the shock-like pain of trigeminal neuralgia. There is some evidence, in three crossover studies (47-49), that different subjects may respond to one antidepressant and not the other, making the trial-and-error use of different drugs within this class a reasonable clinical practice.

It appears that a tricyclic structure is important for antidepressant analgesia, given the lack of effect of the non-TCA mianserin and the lesser effect of the tetracyclic noradrenergic antidepressant maprotiline, compared with the noradrenergic TCAs desipramine and nortriptyline, which appear equal to amitriptyline in efficacy for pain relief. Structure-activity considerations may not necessarily assist in identifying more effective agents. Demethylation of the side chain seems to be important in reducing side effects, as seen with amitriptyline and its demethylated metabolite nortriptyline (49). It is of interest that the tricyclic-based structure carbamazepine, although very effective for the shock-like NP of trigeminal neuralgia, is not very effective or not useful at all for other types of NP. From a structural perspective at the molecular level, the planar structure of TCAs may have some flexibility, allowing the molecule to assume multiple conformations and thereby engage several different pharmacological actions (ie, interact with several transporters, receptors and ion channels). Tetracyclic structures may have more structural rigidity and a lesser ability to interact with several molecular sites. While this may be beneficial for the side effect profile, it might sacrifice actions that actually contribute to efficacy. Furthermore, it may be that a drug that exhibits a multiplicity of actions that affect several aspects of perturbed pain pathways can result in 
synergistic or additive effects, and these are lost with the more specific agents. The concept of polypharmacy or combination therapy using different agents for NP has been addressed directly (65). It may be that the more one strives for a highly selective agent, the more one may sacrifice some of the actions that are contributing to efficacy. We have no way of knowing how individual opioids and anticonvulsants compare because there are no head-to-head trials that compare them. There are some long-term observational data that suggest oxycodone is 'most used' and 'most preferred' over other opioids $(53,54)$.

\section{Why is there partial efficacy with all drugs to date?}

It is not clear why all drugs proven by RCT to exhibit significant pain-relieving effects on NP are only moderately effective, but there are several relevant considerations. One potential reason for limited responses is that damage to or perturbation of the nervous system (peripheral or central) may be so well established in these conditions that the system is only amenable to partial restoration of function (66). A second potential reason is that not enough aspects of the mechanisms that drive the pain are being affected by the drugs used. Multiple mechanisms (both peripheral and central) are implicated in chronic NP, both within an individual and between individuals, and this complexity may be prominently involved in generating and maintaining the pain. Perhaps more attention should be paid to quieting the peripheral input to settle the resultant secondary sensitization that occurs in the central nervous system, as recent cases suggest (67-69). In this regard, existing and improved topical analgesics (70) might be considered as adjuvant therapies along with systemically active agents. A third reason to consider is the multidimensional nature of the chronic pain experience. Thus, pain is a complex experience and has well-recognized sensory, affective and cognitive aspects. In this context, any approach using drugs that target only sensory aspects of chronic pain may only be capable of eliciting a partial response. The developing literature on fibromyalgia may be useful to consider in this regard. While this condition is not necessarily regarded as NP (it lacks an explanation of how 'damage' to the peripheral or central nervous system occurs), it functionally exhibits peripheral and central sensitization in common with other forms of NP and, importantly, responds to drug classes that are used to treat NP (71). Current treatment guidelines recommend multimodal approaches to treatment such as pharmacotherapy, exercise, education, counselling and other psychological approaches, thus combining medical management as well as self-management strategies $(72,73)$. Perhaps the therapy of more classic NP conditions, in which damage to

\section{REFERENCES}

1. Angell M. The Truth About the Drug Companies: How They Deceive Us and What To Do About It. New York: Random House, 2004:240-1.

2. Katz J, Finnerup NB, Dworkin RH. Clinical trial outcome in neuropathic pain; relationship to study characteristics. Neurology 2008;70:263-72.

3. Merskey H, Bodguk H. Classification of Chronic Pain: Description of Chronic Pain Syndromes and Definition of Pain Terms, 2nd edn. Seattle: IASP Press, 1994.

4. Treede RD, Jensen TS, Campbell JN, et al. Neuropathic pain: Redefinition and a grading system for clinical and research purposes. Neurology 2008; $70: 1630-5$.

5. Irving GA. Contemporary assessment and management of neuropathic pain. Neurology 2005;64(12 Suppl 3):S21-7. the central and peripheral nervous system is known to be involved, needs to consider multimodal treatment strategies as well as combinations of drugs with different pharmacodynamics for more effective management. Because of the inadequacies of monotherapy for many patients with severe NP, and because no magic bullet seems imminent, head-to-head RCTs of combinations of drugs useful for NP appear to be important. Head-to-head trials have limitations regarding the evaluation of efficacy if a placebo is not used and the drugs are equal; this type of trial may require more statistical power to show a difference. Limitations of the present review are listed in trial exclusion criteria (see the Methods section) in that the focus is on oral drugs and some NP conditions were excluded. Because of the dearth of head-to-head RCTs in NP and the inadequacies of some of those described here, we clearly need more and improved studies of this nature so that clinicians, grappling with these difficult problems, will know whether newer and more expensive drugs are superior to or comparable with a standard therapy in terms of efficacy, and whether they have fewer significant and intolerable side effects. Investigators designing RCTs of any sort in NP would be wise to follow the Initiative on Methods, Measurement, and Pain Assessment in Clinical Trials guidelines for chronic pain trials (73). Regulatory bodies such as the FDA and Health Canada should require comparative data to be part of the drug approval process. In any trial, principal investigators should have a major role in study design, data analysis and writing the article, and should insist on submission for publication. In the future, clinical trial registries will help to track the course of an RCT and whether it is published. Access to trials submitted for new drug approval are available on the FDA website (www.fda.gov) if submitted for drug approval and unpublished.

DISCLOSURES: Dr Watson has collaborated on clinical trials with Purdue Pharma (Canada) and received reimbursement for presentations by Pfizer Canada Inc and Merck-Frosst Canada Ltd. Dr Gilron has received research support from Pfizer, sanofi-aventis Canada Inc, Novopharm Ltd (Canada), PharmaScience Inc (Canada) and Apotex Inc (Canada), and he has received honoraria for consultations and speaker fees for educational presentations from Pfizer, Merck-Frosst, Johnson \& Johnson Inc (Canada), Ortho-McNeil (USA) and Janssen-Ortho (Canada). Dr Sawynok holds intellectual property licensed to EpiCept Corp (USA) and has received a research grant from this source. She has also had small-scale pharmaceutical industry grant support from Gensia Sicor Pharmaceuticals Inc (USA), Proctor \& Gamble (USA) and Abbott Laboratories (USA).

6. Woolf CJ, Mannion RJ. Neuropathic pain: Aetiology, symptoms, mechanisms and management. Lancet 1999;354:953-4.

7. Merskey H, Hester RA. The treatment of pain with psychotropic drugs. Postgrad Med J 1972;48:594-8.

8. Woodforde JM, Dwyer B, McEwen BW, et al. The treatment of postherpetic neuralgia. Med J Aust 1965;2:869-72.

9. Taub A. Relief of postherpetic neuralgia with psychotropic drugs. J Neurosurgery 1973;39:235-9.

10. Taub A, Collins WF Jr. Observations on the treatment of denervation dysesthesia with psychotropic drugs: Postherpetic neuralgia, anesthesia dolorosa, peripheral neuropathy. In: Bonica JJ, ed. Advances in Neurology, volume 4. 1974:309-15. 
11. Finnerup NB, Otto M, McQuay HJ, et al. Algorithm for neuropathic pain treatment: An evidence-based proposal. Pain 2005;118:289-305.

12. Watson CPN. External validity of pharmaceutical trials in neuropathic pain. In: Rothwell PM, ed. Treating Individuals: From Randomised Trials to Personalised Medicine. Philadelphia: Elsevier, 2007:121-30.

13. Cook RJ, Sackett DL. The number needed to treat: A clinically useful measurement of treatment effect. BMJ 1995;310:452-4.

14. Max MB, Schafer SC, Culnane M, et al. Amitriptyline but not lorazepam relieves postherpetic neuralgia. Neurology 1988:38:1427-32.

15. Jadad AR, Moore A, Carroll D, et al. Assessing the quality of reports of randomized controlled trials: Is blinding necessary? Control Clin Trials 1996;17:1-12.

16. Watson CPN, Evans RJ. A comparative trial of amitriptyline and zimelidine in postherpetic neuralgia. Pain 1985;232:387-94.

17. Gomez-Perez FJ, Rull JA, Dies H, et al. Nortriptyline and fluphenazine in the symptomatic treatment of diabetic neuropathy. A double-blind cross-over study. Pain 1985;23:395-400.

18. Ventafridda V, Caraceni A, Saita L. Trazodone for deafferentation pain. Comparison with amitriptyline. Psychopharmacology 1988;95:S44-S49.

19. Harke H, Gretenkort P, Ladleif HU, et al. The response of neuropathic pain and pain in complex regional pain syndrome I to carbamazepine and sustained relief morphine in patients pre-treated with spinal cord stimulation: A double-blinded randomized study. Anaesth Analg 2001;92:488-95.

20. Pandey CK, Raza R, Tripathi M, et al. The comparative evaluation of gabapentin and carbamazepine for pain management in Guillain-Barre patients in the intensive care unit. Anesth Analg 2005;101:220-5.

21. Galer BS, Lee D, Ma T. Morphidex (morphine sulfate/ dextromethorphan hydrobromide combination) in the treatment of chronic pain: Three multi-center, randomized, double-blind, controlled trials fail to demonstrate enhanced opioid analgesia or reduction in tolerance. Pain 2005;115:284-95.

22. Lynch ME, Clark AJ, Sawynok J. Topical amitriptyline and $1 \%$ ketamine in neuropathic pain syndromes. Anaesthesiology 2005;103:140-6.

23. Keskinbora K, Pekel AF, Aydinli I. Gabapentin and an opioid combination versus opioid alone in the management of neuropathic cancer pain: A randomized trial. J Pain Symptom Manage 2007;34:183-9.

24. Rowbotham MC, Reisner-Keller IA, Fields H. Both intravenous lidocaine and morphine relieve the pain of postherpetic neuralgia. Neurology 1991;41:1024-8.

25. Kok-Yuen H, Huh BK, White WD. Topical amitriptyline versus lidocaine in the treatment of neuropathic pain. Clin J Pain 2008;24:51-5.

26. Pfizer protocol 1008-040. Unpublished data on FDA website. $<$ www.accessdata.fda.gov/scripts/cder/drugsatfda $>$ (Accessed on June 4, 2010).

27. Leijon G, Boivie J. Central post-stroke pain: A controlled trial of amitriptyline and carbamazepine. Pain 1989;36:27-36.

28. Kieburtz K, Simpson D, Yannoutos C. A randomized trial of amitriptyline and mexiletine for painful neuropathy in HIV infection. Neurology 1998;51:1683-9.

29. Morello CM, Leckban SG, Stone CP, et al. Randomized double-blind study comparing the efficacy of gabapentin with amitriptyline on diabetic peripheral neuropathy pain. Arch Int Med 1999;159:1931-7.

30. Graff-Radford SB, Shaw LR, Nalboff BN. Amitriptyline and fluphenazine in postherpetic neuralgia. Clin J Pain 2000;16:188-92.

31. Simpson DA. Gabapentin and venlafaxine for the treatment of painful diabetic neuropathy. J Clin Neuromusc Dis 2001;3:53-62.

32. Raja SN, Haythornethwaite JA, Pappagallo M, et al. Opioids versus antidepressants in postherpetic neuralgia: A randomized placebocontrolled trial. Neurology 2002;59:1015-21.

33. Gilron I, Bailey JM, Tu D, et al. Morphine, gabapentin, or their combination for neuropathic pain. N Engl J Med 2005;352:1324-34.

34. Chandra K, Shafiq N, Pandhi P, et al. Gabapentin versus nortriptyline in postherpetic neuralgia patients: A randomized, double-blind clinical trial - The GONIP trial. Int J Clin Pharm Ther 2006;44:358-63.
35. Khoromi S, Lihong C, Nacker SL, et al. Morphine, nortriptyline and their combination versus placebo in patients with chronic lumbar root pain. Pain 2007;130:66-75.

36. Rintala DH, Holmes SA, Coutade D, et al. Comparison of the effectiveness of amitriptyline and gabapentin on chronic neuropathic pain in persons with spinal cord injury. Arch Phys Med Rehabil 2007;88:1547-60.

37. Jose VM, Bhansali A, Hota D, et al. Randomized double-blind study comparing the efficacy and safety of lamotrigine and amitriptyline in painful diabetic neuropathy. Diabet Med 2007;24:377-83.

38. Frank B, Serpell MG, Hughes J, et al. Comparison of analgesic effects and tolerability of nabilone and dihydrocodeine for chronic neuropathic pain: Randomized, crossover, double-blind study. BMJ 2008;336:199-201.

39. Wu CL, Agarwal S, Prabhav KT, et al. Morphine versus mexiletine for post-amputation pain. Anesthesiology 2008;109:289-96.

40. Hanna M, O'Brien C, Wilson M. Prolonged release oxycodone enhances the effects of existing gabapentin therapy in painful diabetic neuropathy patients. Eur J Pain 2008;12:804-13.

41. Gilron I, Bailey JM, Tu D, Holden RR, Jackson AC, Houlden RL. Nortriptyline and gabapentin, alone and in combination for neuropathic pain: A double-blind, randomised controlled crossover trial. Lancet 2009;374:1252-61.

42. Panerai AE, Monza G, Movilia P, Bianchi M, Francucci BM, Tiengo M. A randomized, within-patient, cross-over, placebocontrolled trial on the efficacy and tolerability of the tricyclic antidepressants chlorimipramine and nortriptyline in central pain. Acta Neurol Scand 1990;82:34-8.

43. Sindrup SH, Gram LF, Brosen K, et al. Selective serotonin reuptake inhibitor paroxetine is effective in the treatment of diabetic neuropathy symptoms. Pain 1990;42:135-44.

44. Sindrup SH, Gram LF, Skjold T. Clomipramine vs desipramine vs placebo in the treatment of diabetic neuropathy symptoms: A double-blind crossover study. Br J Clin Pharmacol 1990;30:683-91.

45. Sindrup SH, Tuxen C, Gram LF. Lack of effect of mianserin on the symptoms of diabetic neuropathy. Clin Pharmacol 1992;43:251-5.

46. Max MB, Lynch SA, Muir J, et al. Effects of desipramine, amitriptyline, and fluoxetine on pain in diabetic neuropathy. N Engl J Med 1992:326:1250-6.

47. Watson CPN, Chipman M, Reed K, et al. Amitriptyline versus maprotiline in postherpetic neuralgia: A randomized double-blind crossover trial. Pain 1992;48:29-36.

48. Vrethem M, Boivie J, Arnquist $\mathrm{H}$, et al. A comparison of amitriptyline and maprotiline in the treatment of painful diabetic neuropathy in diabetics and non-diabetics. Clin J Pain 1997;12:313-23.

49. Watson CPN, Vernich L, Chipman M, et al. Amitriptyline versus nortriptyline in postherpetic neuralgia. Neurology 1998;51:1166-71.

50. Sindrup SH, Bach SW, Madsen MD, et al. Venlafaxine versus imipramine in painful polyneuropathy: A randomized controlled trial. Neurology 2003;60:1284-9.

51. Rowbotham MC, Reisner LA, Davies PS, et al. Treatment response in antidepressant-naïve postherpetic neuralgia patients: Double-blind randomized trial. J Pain 2005;6:741-6.

52. Fudin F, Audette CM. Gabapentin versus amitriptyline for the treatment of peripheral neuropathy. Arch Int Med 2000;160:1040.

53. Watson CPN, Watt-Watson JH, Chipman M. Chronic non-cancer pain and the long-term effects of opioids. Pain Res Manage 2003;9:19-24.

54. Watson CPN, Watt-Watson JH, Chipman M. The long-term safety and efficacy of opioids: A survey of 84 selected patients with chronic noncancer pain. Pain Res Manage 2010. (In press)

55. Gutstein HB, Akil H. Opioid analgesics. In: Hardman JG, Limbird LE, eds. Goodman and Gilman's The Pharmacological Basis of Therapeutics, 11th edn. New York: McGraw-Hill, 2006:547-90.

56. Baillie JK, Power I. The mechanism of action of gabapentin in neuropathic pain. Curr Opin Invest Drugs 2006;71:33-9.

57. Tanabe M, Takasu K, Takeuchi Y, Ono H. Pain relief by gabapentin and pregabalin via supraspinal mechanisms after peripheral nerve injury. J Neurosci Res 2008;86:3258-64.

58. Baldessarini RJ. Drugs and the treatment of psychiatric disorders: Depression and anxiety disorders. In: Hardman JG, Limbird LE, eds. Goodman and Gilman's The Pharmacological Basis of Therapeutics, 11th edn. New York: McGraw-Hill, 2006:437.

59. Mico JA, Ardid D, Berrocoso E, et al. Antidepressants and pain. Trends Pharmacol Sci 2006;27:348-54. 
60. Banks SM, Kerns RD. Explaining high rates of depression in chronic pain: A diathesis-stress framework. Psychol Bull 1996;119:95-110.

61. Blackburn-Munro G, Blackburn-Munroe RE. Chronic pain, chronic stress and depression: Coincidence or consequence? J Endocrinol 2001;13:1009-23.

62. Watson CPN, Evans RJ, Reed K, et al. Amitriptyline versus placebo in postherpetic neuralgia. Neurology 1982;32:671-3.

63. Onghena P, Van Houdenhove B. Antidepressant-induced analgesia in chronic non-malignant pain: A meta-analysis of 39 placebo controlled studies. Pain 1992;49:205-19.

64. Benarroch EE. Descending monoaminergic pain modulation. Neurology 2008;71:217-21.

65. Gilron I, Max MB. Combination pharmacotherapy for neuropathic pain: Current evidence and future directions. Exp Opin Neurotherapeutics 2005;5:823-30

66. Watson CPN, Morshead C, Van der Kooy D, Deck J, Evans RJ. Post-herpetic neuralgia: Post-mortem analysis of a case. Pain 1988;34:129-38.
67. Petersen KL, Rice FL, Suess F, et al. Relief of postherpetic neuralgia by resection of painful skin. Pain 2002;98:119-26.

68. Petersen KL, Rowbotham MC. Relief of postherpetic neuralgia by resection of painful skin: 5 years later. Pain 2007;131:214-8.

69. Watson CPN, Stinson J, Dostrovsky JO, et al. Nerve relocation may relieve causalgia: A case report. Pain 2007;132:211-7.

70. Watson CPN. Topical agents for neuropathic pain. A systematic review. In: Merskey H, Loeser JD, Dubner R, eds. The Paths of Pain. Seattle: IASP Press, 2005.

71. Carville SF, Arendt-Nielsen S, Blindel H, et al. EULAR evidencebased recommendations for the management of fibromyalgia syndrome. Ann Rheum Dis 2008;67:536-46.

72. Lawson K. Pharmacological treatment of fibromyalgia: Do complex conditions need complex therapies? Drug Discov Today 2008;13:333-40.

73. Dworkin RH, Turk DC, Peirce-Sandner F, et al. Research Design Considerations for Confirmatory Chronic Pain Clinical Trials "IMMPACT Recommendations." Pain 2010;149:177-93. 


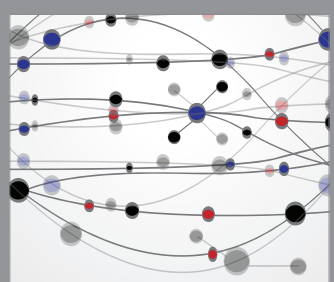

The Scientific World Journal
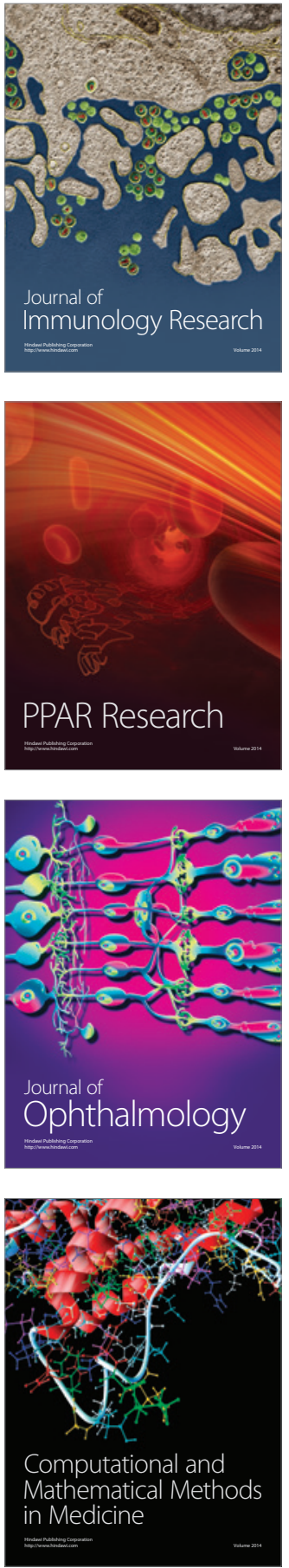

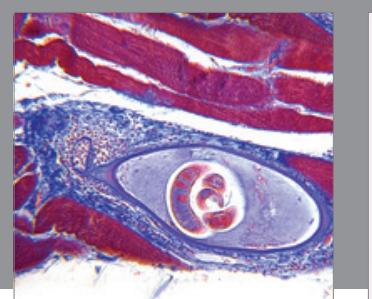

Gastroenterology Research and Practice

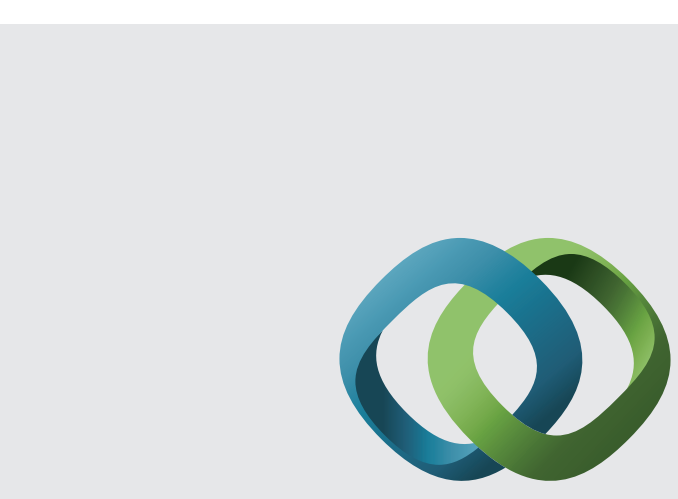

\section{Hindawi}

Submit your manuscripts at

http://www.hindawi.com
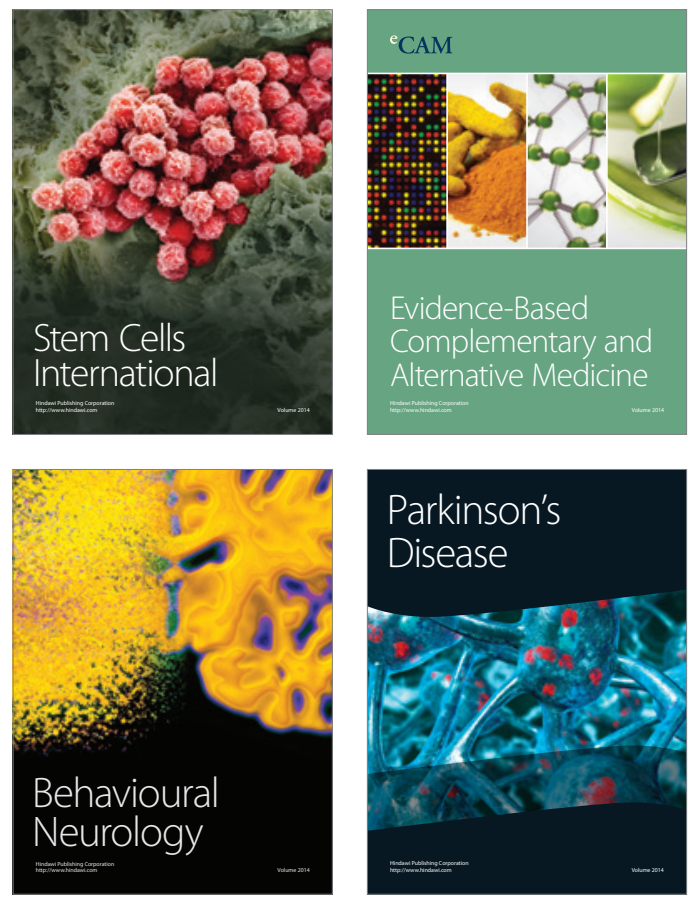
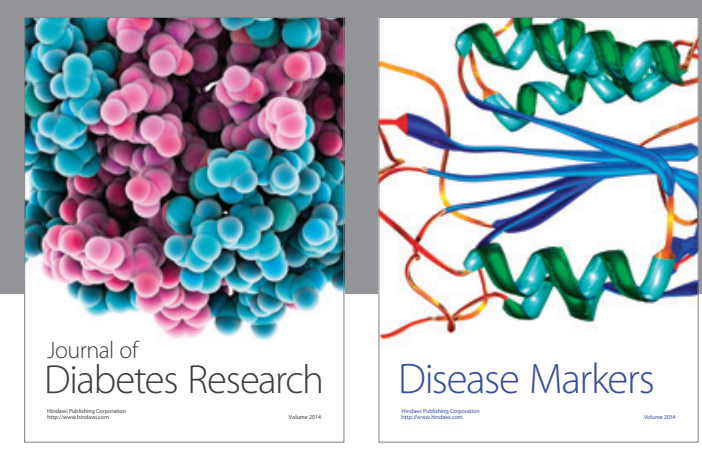

Disease Markers
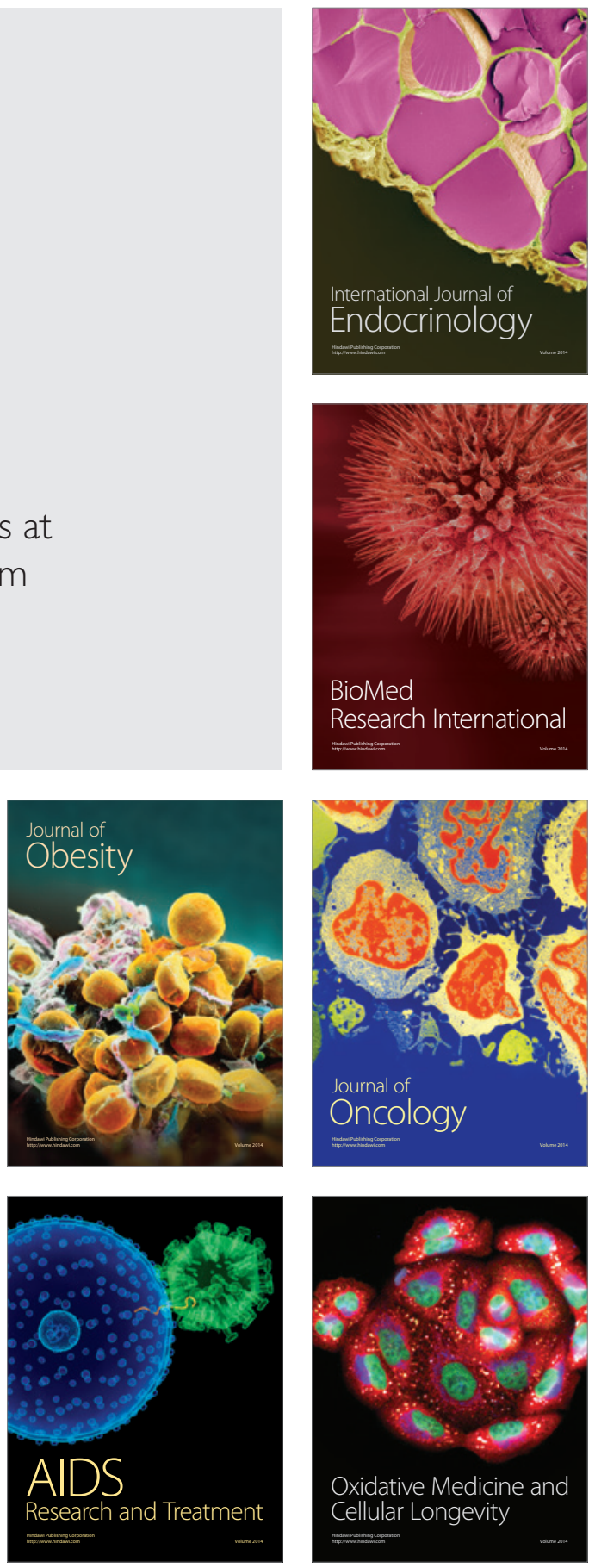